\title{
Global existence of solution for multidimensional generalized double dispersion equation
}

Xiaoqiang Dai ${ }^{\text {* }}$

\section{"Correspondence:}

daixiaoqiang@just.edu.cn

'Department of Electronic

Information, Jiangsu University of

Science and Technology, Zhenjiang,

People's Republic of China

\begin{abstract}
In this paper, we study the Cauchy problem of multidimensional generalized double dispersion equation. To prove the global existence of solutions, we introduce some new methods and ideas, and fill some gaps in the established results.
\end{abstract}

MSC: 35Q53

Keywords: Multidimensional generalized double dispersion equation; Cauchy problem; Global solution; Existence

\section{Introduction}

In this paper, we study the Cauchy problem of the multidimensional generalized double dispersion equation

$$
\begin{aligned}
& u_{t t}-\Delta u-\Delta u_{t t}+\Delta^{2} u-k \Delta u_{t}=\Delta f(u), \quad x \in \mathbb{R}^{n}, t>0, \\
& u(x, 0)=u_{0}(x), \quad u_{t}(x, 0)=u_{1}(x), \quad x \in \mathbb{R}^{n},
\end{aligned}
$$

where $k$ is a constant. Equation (1.1) is called the generalized damped multidimensional Boussinesq equation in [1], which is related to some Boussinesq equations and their generalized forms. Therefore we may consider this class of equations as an approach for propagation of long waves on shallow water; see [1-12] and references therein. Thus, in the present paper, we call Eq. (1.1) the multidimensional generalized double dispersion equation, since in the one-dimensional case, Eq. (1.1) is the generalized double dispersion equation

$$
u_{t t}-u_{x x}-u_{x x t t}+u_{x x x x}-k u_{x x t}=f(u)_{x x}
$$

which was introduced to consider the model of interaction between the surface of a nonlinear elastic rod, whose material is hyperelastic (e.g., the Murnaghan material). For derivation and research background, we refer the readers to [3-12] and references therein. When $f^{\prime}(u)$ is bounded below, the initial boundary value problem and the Cauchy problem of Eq. (1.3) were considered in [5] and [6], respectively. Later the corresponding initial boundary value problem was studied in [3], where $f(u)$ is either of general convex form or

(c) The Author(s) 2019. This article is distributed under the terms of the Creative Commons Attribution 4.0 International License (http://creativecommons.org/licenses/by/4.0/), which permits unrestricted use, distribution, and reproduction in any medium, provided you give appropriate credit to the original author(s) and the source, provide a link to the Creative Commons license, and indicate if changes were made. 
the particular case $f(u)=|u|^{p}, p>1$. These conditions satisfied by $f(u)$ enable authors to give some sharp conditions on the global existence for the initial boundary value problem of Eq. (1.3) and some invariant sets by the variational principle. However, for the multidimensional generalized double dispersion equation, it was still an open question of giving the local and global well-posedness before the publication of [1]. In [1] the authors managed to deal with the Cauchy problem of the multidimensional nonlinear evolution equation (1.1)-(1.2). They first gave the existence of local solutions. By some estimates of a local solution they obtained the existence of a global solution, even though some gaps are still there, for example, it is not clear how to define an appropriate space that contains $\left(u_{t t}, u_{t}\right)$, which also needs additional restrictions on other parameters like $s$. Also, in the proof of [1, Lemma 3.3] the inequality $\left\|u^{\rho}\right\|_{\infty} \leq C\|\Delta u\|$ does not hold if $2 \leq n \leq 4$. Moreover, the energy also needs further estimation. Despite these gaps, the work of [1] motivated many studies like [2-13].

So in the present paper, we provide more insight into overcoming the difficulties in the multidimensional case and fill the gaps in [1] to obtain the global existence of a solution for problem (1.1)-(1.2).

In this paper, we use same notations as in [1] and denote $\|\cdot\|_{2}$ by $\|\cdot\|$. Considering problem (1.1)-(1.2), as in [1, Theorem 2.1], we present the following proposition.

Propostion $1.1([1])$ Let $f(u) \in C^{[s]+1}(\mathbb{R})$ for some $s>\frac{n}{2}$. Then for any $u_{0} \in H^{s}$ and $u_{1} \in H^{s-1}$, problem (1.1)-(1.2) admits a unique local solution $u(t) \in C\left(\left[0, T^{0}\right) ; H^{s}\right) \cap$ $C^{1}\left(\left[0, T^{0}\right) ; H^{s-1}\right)$ with $u_{t t} \in C\left(\left[0, T^{0}\right) ; H^{s-2}\right)$, where $T^{0}$ is the maximal existence time of $u(t)$. Moreover, if

$$
\sup _{t \in\left[0, T_{0}\right)}\left(\|u(t)\|_{H^{s}}+\left\|u_{t}(t)\right\|_{H^{s-1}}\right)<\infty
$$

then $T^{0}=+\infty$.

\section{Existence of global solution}

In this section, we give two global existence theorems for problem (1.1)-(1.2). First, let us give some preliminary lemmas.

Lemma 2.1 Let the assumptions in Proposition 1.1 hold. Assume that $s \geq \frac{3}{2},(-\Delta)^{-\frac{1}{2}} u_{1} \in$ $L^{2}$, and $F\left(u_{0}\right) \in L^{1}$. Then for the solution $u$ given in Proposition 1.1, we have

$$
E(t)+k \int_{0}^{t}\left\|u_{\tau}\right\|^{2} \mathrm{~d} \tau=E(0), \quad 0 \leq t<T^{0}
$$

where

$$
E(t)=\frac{1}{2}\left(\left\|(-\Delta)^{-\frac{1}{2}} u_{t}\right\|^{2}+\left\|u_{t}\right\|^{2}+\|u\|^{2}+\|\nabla u\|^{2}\right)+\int_{\mathbb{R}^{n}} F(u) \mathrm{d} x .
$$

Lemma 2.2 Let the assumptions of Lemma 2.1 hold. Assume that $k \geq 0$ and that either $F(u) \geq 0$ for all $u \in \mathbb{R}$ or $f(0)=0$ and $\inf _{u \in \mathbb{R}^{n}} f^{\prime}(u)=C_{0}>-1$. Then for the solution $u$ given 
in Proposition 1.1, we have

$$
\left\|(-\Delta)^{-\frac{1}{2}} u_{t}\right\|^{2}+\left\|u_{t}\right\|^{2}+\|u\|^{2}+\|\nabla u\|^{2} \leq C E(0), \quad 0 \leq t<T^{0},
$$

where $C \geq 2$ is a positive constant.

Proof

(i) If $k \geq 0$ and $F(u) \geq 0$, then (2.2) immediately follows from (2.1).

(ii) If $k \geq 0, f(0)=0$, and $\inf _{u \in \mathbb{R}^{n}} f^{\prime}(u)=C_{0}>-1$, then letting $k_{0}=\max \left\{-C_{0}, 0\right\}$ and $f_{1}(u)=f(u)+k_{0} u$, we have $0 \leq k_{0}<1, f_{1}(0)=0$, and $f_{1}^{\prime}(u) \geq 0$. Hence $F_{1}(u)=\int_{0}^{u} f_{1}(\tau) \mathrm{d} \tau \geq 0$. Substituting $\int_{\mathbb{R}^{n}} F(u) \mathrm{d} x=\int_{\mathbb{R}^{n}} F_{1}(u) \mathrm{d} x-\frac{k_{0}}{2}\|u\|^{2}$ into (2.1) yields (2.2) with $C=\frac{2}{1-k_{0}}$.

Based on Lemma 2.2, the following two theorems show the existence of global solution for problem (1.1)-(1.2).

Theorem 2.3 Let $n=1,2, f(u) \in C^{2}(\mathbb{R}), u_{0} \in H^{\frac{3}{2}}, u_{1} \in H^{\frac{1}{2}},(-\Delta)^{-\frac{1}{2}} u_{1} \in L^{2}$, and $F\left(u_{0}\right) \in L^{1}$. Assume that $k \geq 0$ and that either $F(u) \geq 0$ for any $u \in \mathbb{R}$ or $f(0)=0$ and $\inf _{u \in \mathbb{R}^{n}} f^{\prime}(u)>$ -1 . Then problem (1.1)-(1.2) admits a unique global solution $u(t) \in C\left([0, \infty) ; H^{1}\right) \cap$ $C^{1}\left([0, \infty) ; L^{2}\right)$.

Proof Setting $s=\frac{3}{2}$, by Proposition 1.1 we have $\frac{3}{2}>\frac{n}{2}$ for $n=1,2$. Hence by Proposition 1.1 problem (1.1)-(1.2) admits a unique local solution $u \in C\left(\left[0, T^{0}\right) ; H^{\frac{3}{2}}\right) \cap C^{1}\left(\left[0, T^{0}\right) ; H^{\frac{1}{2}}\right) \subset$ $C\left(\left[0, T^{0}\right) ; H^{1}\right) \cap C^{1}\left(\left[0, T^{0}\right) ; L^{2}\right)$. Furthermore, from Lemma 2.2 we get

$$
\sup _{t \in\left[0, T_{0}\right)}\left(\|u(t)\|_{H^{1}}+\left\|u_{t}(t)\right\|_{L^{2}}\right)<\infty
$$

which, together with Proposition 1.1, gives $T^{0}=+\infty$.

Theorem 2.4 Let $n=3$ and $s>\frac{3}{2}$ or $n=4$ and $s>2$, and let $f(u) \in C^{[s]+1}(\mathbb{R}), u_{0} \in H^{s}$, $u_{1} \in H^{s-1},(-\Delta)^{-\frac{1}{2}} u_{1} \in L^{2}$, and $F\left(u_{0}\right) \in L^{1}$. Assume that $k \geq 0$ and that either $F(u) \geq 0$ for any $u \in \mathbb{R}$ or $f(0)=0$ and $\inf _{u \in \mathbb{R}^{n}} f^{\prime}(u)>-1$. Then problem (1.1)-(1.2) admits a unique global solution $u(t) \in C\left([0, \infty) ; H^{1}\right) \cap C^{1}\left([0, \infty) ; L^{2}\right)$.

Proof First, we have $s>\frac{n}{2}$ for $n=3$, 4. Hence by Proposition 1.1 problem (1.1)-(1.2) admits a unique local solution $u \in C\left(\left[0, T^{0}\right) ; H^{s}\right) \cap C^{1}\left(\left[0, T^{0}\right) ; H^{s-1}\right) \subset C\left(\left[0, T^{0}\right) ; H^{1}\right) \cap$ $C^{1}\left(\left[0, T^{0}\right) ; L^{2}\right)$. The remainder of this proof is the same as that in the proof of Theorem 2.3.

In light of the argument developed in the present paper, we can conclude that for problem (1.1)-(1.2), if we adopt the course in [1], that is, first prove the existence of a local solution and then get the existence of a global solution, then we can only obtain the existence of global $H^{1}$ solutions. Moreover, as in Theorem 2.3 and Theorem 2.4, we see that to obtain the global existence of an $H^{1}$ solution from the existence of a local solution, it is reasonable to assume that $u_{0} \in H^{s}$ for some $s \geq \frac{3}{2}$. Namely, our argument relies on the assumption of a higher-order regularity of the initial data than that of the corresponding solution. Obviously, we cannot get the same regularity for both the initial data and the corresponding solution, which is still an open question for further investigation. 
Acknowledgements

The author thanks the referees for their constructive suggestions.

\section{Funding}

This work was supported by the Natural Science Foundation of Jiangsu Province (BK20160564)

\section{Abbreviations}

Not applicable.

Availability of data and materials

Not applicable.

\section{Competing interests}

The author declares that there are no competing interests.

\section{Authors' contributions}

The sole author completed the paper and approved the final manuscript.

\section{Publisher's Note}

Springer Nature remains neutral with regard to jurisdictional claims in published maps and institutional affiliations.

Received: 26 August 2019 Accepted: 24 September 2019 Published online: 01 October 2019

\section{References}

1. Polat, N., Ertaş, A.: Existence and blow-up of solution of Cauchy problem for the generalized damped multidimensional Boussinesq equation. J. Math. Anal. Appl. 349(1), 10-20 (2009)

2. Liu, Y., Xu, R.: Global existence and blow up of solutions for Cauchy problem of generalized Boussinesq equation. Phys. D, Nonlinear Phenom. 237(6), 721-731 (2008)

3. Liu, Y., Xu, R.: Potential well method for Cauchy problem of generalized double dispersion equations. J. Math. Anal. Appl. 338(2), 1169-1187 (2008)

4. Lian, W., Xu, R.: Global well-posedness of nonlinear wave equation with weak and strong damping terms and logarithmic source term. Adv. Nonlinear Anal. 9(1), 613-632 (2020)

5. Chen, G., Wang, Y., Wang, S.: Initial boundary value problem of the generalized cubic double dispersion equation. J. Math. Anal. Appl. 299(2), 563-577 (2004)

6. Wang, S., Chen, G.: Cauchy problem of the generalized double dispersion equation. Nonlinear Anal., 64(1), 159-173 (2006)

7. Xu, R., Liu, Y.: Global existence and nonexistence of solution for Cauchy problem of multidimensional double dispersion equations. J. Math. Anal. Appl. 359(2), 739-751 (2009)

8. Xu, R., Liu, Y., Liu, B.: The Cauchy problem for a class of the multidimensional Boussinesq-type equation. Nonlinear Anal. 74(6), 2425-2437 (2011)

9. Polat, N., Piskin, E.: Asymptotic behavior of a solution of the Cauchy problem for the generalized damped multidimensional Boussinesq equation. Appl. Math. Lett. 25(11), 1871-1874 (2012)

10. Wang, H., Wang, S.: Global existence and asymptotic behavior of solution for the Rosenau equation with hydrodynamical damped term. J. Math. Anal. Appl. 401(2), 763-773 (2013)

11. Wang, S., Da, F.: On the asymptotic behaviour of solution for the generalized double dispersion equation. Appl. Anal. 92(6), 1179-1193 (2013)

12. Wang, Y., Wei, C.: Asymptotic profile of global solutions to the generalized double dispersion equation via the nonlinear term. Z. Angew. Math. Phys. 69(2), 34 (2018)

13. Saanouni, T.: Global well-posedness of some high-order focusing semilinear evolution equations with exponential nonlinearity. Adv. Nonlinear Anal. 7(1),67-84 (2017)

\section{Submit your manuscript to a SpringerOpen ${ }^{\circ}$ journal and benefit from:}

- Convenient online submission

- Rigorous peer review

- Open access: articles freely available online

- High visibility within the field

- Retaining the copyright to your article

Submit your next manuscript at $\boldsymbol{~ s p r i n g e r o p e n . c o m ~}$ 